\title{
A Micro-mechanics Model for Imperfect Interface in Dielectric Materials
}

\author{
H. Fan \\ School of Mechanical and Production Engineering \\ Nanyang Technological University \\ Singapore 639798, Republic of Singapore \\ K.Y. Sze \\ Department of Mechanical Engineering \\ The University of Hong Kong \\ Hong Kong SAR, P.R. China
}

\begin{abstract}
The interface between two dielectric bodies is considered imperfect if there are defects (micro-voids and micro cracks) present on the interface. For such interface, the perfect continuity condition across the interface is no longer valid and its use in analysis becomes questionable. To account for this imperfection, we propose a micro-mechanics model based on self-consistent scheme, leading to the establishment of a constitutive relationship between the electric displacement and potential discontinuity across the imperfect interface.
\end{abstract}

Key words: Interface, crack, dielectric material, self-consistent scheme 


\section{Introduction}

A perfect interface in dielectric boundary value problems is characterized by the following continuity conditions:

$$
\phi^{+}=\phi^{-}
$$

and

$$
D_{2}^{+}=D_{2}^{-}
$$

along the interface (refer the coordinate system in Fig. 1(a)). Where $\phi$ is the electric potential and $\boldsymbol{D}$ is the electric displacement vector. The superscripts "+" and "-" denote $y=0^{+}$and $y=0^{-}$, respectively.

As depicted in Fig. 1(b), for an interface with micro cracks or voids, the continuity conditions of Eqn.(1.1) and (1.2) are no longer valid. However, if the representative micro-defects size is much smaller than the characteristic dimension of the specimen or the structure, we may merge the micro cracks along the interface into a continuous damaged interface. For analyses at the structure level, the following condition may be assumed

$$
\bar{D}_{2}=k \overline{[\phi]} \quad \text { or } \quad \overline{[\phi]}=\bar{D}_{2} / k
$$

where the bar denotes the average over an area of a scale much greater than the dimension of the micro-cracks. The bracket value of $\phi,[\phi]$, denotes the discontinuity of $\phi$ across the interface, namely,

$$
[\phi]=\phi^{-}-\phi^{+}
$$

where $k$ is dependent on the micro-structure as well as the bi-material properties. For a perfect interface $k$ tends to infinity, while a vanishing $k$ implies the separation of the two bodies. Eqn.(1.3) has been referred to as the "spring model" for damaged interfaces.

A few studies on continuous damaged interface have been reported in the literature. Hashin (1991) applied the spring model for the imperfect interface betwwen inclusion and matrix. The author also complied a list of relevant investigations reported previously. Another group of researchers advocating the interface spring model comes from the non-destructive evaluation society. It has been proved that the interface spring model is easy to use and can explaine a certain phenomena observed from the experiments. The analytical results, quoted for comparision with the testing data, 
were based on either periodically distributed interface cracks (e.g. Margetan et al, 1988) or homogeneous medium due to the mathematical difficulty involved in bi-material interface problem. More related to the present study, Benveniste (1999), Miloh and Benveniste (1999) and Benveniste and Miloh (1999) used "spring" model for the imperfect conductivity interfaces.

The purpose of the present study is to relate the spring constant $k$ of Eqn.(1.3) with micro-cracks or voids along the interface. This relationship is to be sought by applying the so-called "averaging" scheme, which is also known as the micro-mechanics modeling procedure of relating the microstructure(s) with the macro constitutive parameters. Such an approach has been widely used in composite mechanics investigation. In fact, a number of micro-mechanics schemes and models have been developed for estimating effective material properties of the composites, such as elastic modulus, dielectric constants and thermal conductivity to name a few (see Christensen, 1990 for a recent review). In the present study, we apply a generalized self-consistent scheme of composite mechanics to model imperfect interface between two dielectric bodies. In the following sections, we present discussions which are primarily focused on linear dielectric materials. In terms of the mathematical form, they are read as,

$$
\begin{aligned}
D_{i} & =\varepsilon_{i j} E_{j} \\
E_{i} & =-\phi_{, i} \\
\phi_{, i i} & =0
\end{aligned}
$$

where $\boldsymbol{D}, \boldsymbol{E}, \boldsymbol{\varepsilon}$ and $\phi$ are the electric displacement, electric field, permittivity tensor and electric potential respectively. In Eqs.(1.4) to (1.6), the index summation convention is employed.

\section{Self-consistent model for the imperfect interface ---- plane configuration}

This configuration allows us to formulate our model in an analytical form, and highlight the selfconsistent scheme. Furthermore, the solution of the singular integral equations based on the model can be compared with the subsequent finite element analysis results.

A schematic representation of an interface under consideration is given in Fig. 1. On the macro level (Fig. 1(a)) the interface is continuous without discrete micro-defects. The continuity condition across the interface follows the spring model (Eqn.(1.3)). On the micro-level (i.e. the scale of micro-defects), there are randomly distributed cracks along the interface as shown in Fig. 1(b). A generalized self-consistent model can be used to equate Fig. 1(b) with Fig. 1(c). 
Let us divide the interface into three different regions according to the self-consistent scheme as follows:

- Region $-a<x<a$, micro crack

- Regions $-b<x<-a$ and $a<x<b$, two perfectly bonded regions

- Regions $-L<x<-b$ and $b<x<L$, regions applied the averaging procedure whose properties are unknown yet. Note that $a$ and $b$ are much smaller than $L$

The continuity or the boundary conditions of these three regions are different. Over the crack surfaces, i.e. $(-a<x<a)$, we have

$$
D_{2}^{+}=D_{2}^{-}=0
$$

The electric potential function $\phi$ has a discontinuity across the interfacial crack. It should be mentioned that different conditions on the interfacial crack surfaces have been used in analytical analyses (Suo, et al 1992). However, no matter which conditions we adopt, the essence of the generalized self-consistent scheme remains the same.

On the other hand, for the perfectly bonded region ( $-b<x<-a$ and $a<x<b)$, Eqns.(1.1) and (1.2) apply. The effective region ( $-L<x<-b$ and $b<x<L)$ with continuous damage is described by the spring model of Eqn. (1.3).

For the derivation of the governing equations based on the self-consistent micro-mechanics model, a new quantity, the electric potential jump density, $h(x)$, is introduced.

$$
h(x) \equiv \frac{\partial[\phi]}{\partial x}
$$

Over the three regions as defined above, the average potential discontinuity can be written by

$$
\overline{[\phi]}=\frac{1}{2 L} \int_{-L}^{L}[\phi] d x=\frac{1}{2 b} \int_{-a}^{a}[\phi] d x
$$

with $[\phi]=0$ at $x=-a$ and $a$, the above expression may be written as, via integration by part,

$$
\overline{[\phi]}=-\frac{1}{2 b} \int_{-a}^{a} x h(x) d x
$$

By the analogy between the present boundary value problem defined by Eqns.(1.4) to (1.6) and the anti-plane problem in linear elasticity, the governing integral equation is written as : 


$$
\frac{1}{\pi} \int_{-a}^{a} \frac{h(s) d s}{x-s}+\frac{1}{\pi} \int_{-L}^{-b} \frac{h(s) d s}{x-s}+\frac{1}{\pi} \int_{b}^{L} \frac{h(s) d s}{x-s}=-\frac{2 D_{2}^{\infty}}{C}
$$

where

$$
C=\frac{2 \varepsilon^{(1)} \varepsilon^{(2)}}{\varepsilon^{(1)}+\varepsilon^{(2)}}
$$

for isotropic dielectric materials.

By carrying out the integration by part and noting that $a$ and $b$ are much smaller than $L$, we can approximate Eqn. (2.4) as follows.

$$
\int_{-1}^{1} \frac{h(t) d t}{\xi-t}-\frac{\overline{[\phi]}}{b}\left(\frac{1}{1+\rho \xi}-\frac{1}{1-\rho \xi}\right)=-\frac{2 \pi D_{2}^{\infty}}{C}
$$

where $\rho=a / b$. The non-dimensional coordinates are

$$
\xi=x / a \quad \text { and } \quad t=s / a
$$

Eqns.(2.3a) and (2.6) constitute a generalized self-consistent description to the problem. The average electric potential, $\overline{[\phi]}$, is computed from $h(x)$. According to Eqn.(2.3a) however the solution of Eqn. (2.6) (for $h(x)$ ) requires the knowledge of the averaged potential, $\overline{[\phi]}$.

By substituting $\overline{[\phi]}$ given by Eqn.(2.3a) into Eqn.(2.6), we have an integral equation for $h(x)$ with the first term having a Cauchy type kernel and second term with a non-singular kernel. The solution of this singular integral equation can be obtained numerically by using, say, Erdogan's scheme (1975). Before discussing the numerical solution, we first present an analytical approximate solution valid for $\rho<<1$ which is obtained by neglecting interactions among the micro-cracks. The approximate solution of Eqn.(2.6) is found in a closed form,

$$
h(t)=-\frac{2 D_{2}^{\infty}}{C}\left(1-t^{2}\right)^{-1 / 2} t
$$

By combine the above expression with Eqns.(2.2) and (2.3), one has

$$
\overline{[\phi]}=-\frac{a \rho}{2} \int_{-1}^{1} t h(t) d t=\frac{a \pi \rho D_{2}^{\infty}}{2 C} .
$$

Comparing Eqn. (2.8) with Eqn. (1.3), the following result is obtained

$$
k=\frac{2 C}{a \pi} \frac{1}{\rho} .
$$


For interfaces with large $\rho$, a numerical scheme, say Erdogan (1975), is required, with which the solution of whole range of $\rho$ is obtained.

In the present study, instead of using the singular equation approach, we employed finite element method to carry out the numerical calculations. The stability and reliability of the commercial FEM package, ABAQUS (1998), shows great advantage over the solution scheme via Erdogan's (1975) formulation for singular integral equation. It has been noticed that finite element method for solving this kind of mix-boundary value problem has substantial advantage for the problems where the singularities are changing (Fan et al (2000)).

Figure 2 shows the system used for the finite element calculations. The spatial domain consists of two $2 L \times h$ dissimilar dielectric materials with the interface at $y=0$. For the calculation, we fixed certain parameters. They are $b=1, h=L=15$ and $\varepsilon^{(1)}=1$ whereas $a$ and $\varepsilon^{(2)}$ will be varied. The prescribed boundary conditions were: $D_{y}=q=1$ at $y= \pm h$ and $D_{x}=0$ at $x= \pm L$. At the interface, the governing conditions were:

(i) $D_{y}=0$ over the microcrack $(-a<x<a)$

(ii) $[\phi]=0$ over the perfectly bonded region $(-b<x<-a$ and $a<x<b)$

(iii) $k[\phi]=q$ over the effective region $(-L<x<-b$ and $b<x<L)$

and, most importantly,

(iv) $k \overline{[\phi]}=q$ over the microcrack and perfectly bonded region $(-b<x<b)$

Owing to symmetry, only $x \geq 0$ is modeled and the commercial finite element software ABAQUS (1998) is resorted to. While it is straightforward to prescribe (i) and (ii), (iii) for any $k$ can be enforced by using the linear constraint equation facility available in ABAQUS. (iv) can not be prescribed or enforced by any direct means. Thus, we vary $k$ in the linear constraint equation until the following $0.5 \%$ tolerance condition is met:

$$
-0.005<k \overline{[\phi]} / q-1<0.005
$$

where

$$
\overline{[\phi]}=\frac{\int_{-b}^{b}[\phi] d x}{\int_{-b}^{b} d x}=\frac{1}{b} \int_{0}^{b}[\phi] d x=\frac{1}{b} \int_{0}^{a}[\phi] d x
$$


Regarding the finite element mesh, around 10,000 four-node plane elements are employed with the highest element densities arranged at $(x=a, y=0)$ and $(x=b, y=0)$ so that the associated electric flux concentration can be accurately captured. It has been checked by including more elements into the mesh that the latter has been fine enough to secure sufficiently accurate predictions. Table 1 lists the computed $k a / C$, by varying $a / b$ and the permittivity of the material below $y=0$. The results are insensitive to $\varepsilon^{(2)}$ or $\mathrm{C}\left(=2 \varepsilon^{(1)} \varepsilon^{(2)} /\left(\varepsilon^{(1)}+\varepsilon^{(2)}\right)\right)$. The finite element results together with Eqn.(2.9) are shown in Figure 3.

Table 1 : Computed $k a / C$ for plane configuration

\begin{tabular}{|c|ccccccc|}
\hline & \multicolumn{7}{|c|}{$\varepsilon^{(2)}$} \\
\cline { 2 - 8 }$a / b$ & 1 & 5 & 10 & 50 & 100 & 500 & 1000 \\
\hline 0.02 & 35.91 & 35.93 & 35.90 & 35.93 & 35.90 & 35.93 & 35.92 \\
0.032 & 21.45 & 21.45 & 21.44 & 21.45 & 21.43 & 21.43 & 21.44 \\
0.05 & 13.37 & 13.33 & 13.35 & 13.35 & 13.36 & 13.36 & 13.35 \\
0.07 & 9.396 & 9.406 & 9.413 & 9.395 & 9.402 & 9.402 & 9.405 \\
0.1 & 6.494 & 6.522 & 6.494 & 6.497 & 6.499 & 6.498 & 6.496 \\
& & & & & & & \\
0.14 & 4.590 & 4.590 & 4.589 & 4.592 & 4.588 & 4.590 & 4.589 \\
0.2 & 3.175 & 3.166 & 3.165 & 3.163 & 3.165 & 3.165 & 3.164 \\
0.3 & 2.048 & 2.045 & 2.045 & 2.045 & 2.045 & 2.045 & 2.044 \\
0.4 & 1.473 & 1.472 & 1.472 & 1.472 & 1.471 & 1.471 & 1.471 \\
0.5 & 1.111 & 1.111 & 1.111 & 1.111 & 1.112 & 1.112 & 1.111 \\
& & & & & & & \\
0.6 & 0.8571 & 0.8571 & 0.8558 & 0.8550 & 0.8554 & 0.8554 & 0.8551 \\
0.7 & 0.6542 & 0.6542 & 0.6547 & 0.6547 & 0.6544 & 0.6544 & 0.6541 \\
0.8 & 0.4819 & 0.4819 & 0.4819 & 0.4815 & 0.4819 & 0.4819 & 0.4817 \\
0.9 & 0.3125 & 0.3121 & 0.3123 & 0.3120 & 0.3122 & 0.3122 & 0.3121 \\
\hline
\end{tabular}

\section{Three dimensional configuration}

For the three dimensional configuration, the finite element method is again adopted. The finite element model is built upon the assumption that the three-phase-model forms an axial symmetric configuration as shown in Figure 2. The problem parameters, boundary and interfacial conditions are essentially the same as the plane case except that $x$ becomes $r$ and $r$ must be positive. Moreover, the area average of the electric potential discontinuity with $0.5 \%$ tolerance condition in $(2.10)$ has to be amended as: 


$$
\overline{[\phi]}=\frac{\int_{0}^{b} 2 \pi r[\phi] d r}{\int_{0}^{b} 2 \pi r d r}=\frac{2}{b^{2}} \int_{0}^{b} r[\phi] d r=\frac{2}{b^{2}} \int_{0}^{a} r[\phi] d r
$$

Using the same mesh for the plane configuration built by four-node axisymmetric elements, Table 2 lists the computed $k a / C$, by varying $a / b$ and the permittivity of the material below $y=0$. Again, the results are insensitive to $\varepsilon^{(2)}$ or C. Figure 4 shows the $\log -\log$ plot for $k a / C$ against $a / b$. Similar to the Figure 3, the relation is linear for small $a / b$.

Table 2. Computed $k a / C$ for axisymmetric configuration

\begin{tabular}{|c|ccccccc|}
\hline & \multicolumn{7}{|c|}{$\varepsilon^{(2)}$} \\
\cline { 2 - 8 } & 1 & 5 & 10 & 50 & 100 & 500 & 1000 \\
\hline 0.02 & 3530 & 3530 & 3530 & 3531 & 3529 & 3531 & 3531 \\
0.032 & 1290 & 1289 & 1289 & 1289 & 1289 & 1290 & 1290 \\
0.05 & 507.6 & 508.5 & 509.3 & 507.5 & 505.0 & 506.1 & 507.6 \\
0.07 & 253.6 & 253.8 & 253.8 & 253.7 & 253.6 & 253.6 & 253.5 \\
0.1 & 122.0 & 122.4 & 122.2 & 122.6 & 122.3 & 122.2 & 122.4 \\
& & & & & & & \\
0.14 & 61.81 & 61.76 & 61.80 & 61.82 & 61.75 & 61.80 & 61.79 \\
0.2 & 30.08 & 30.00 & 30.05 & 30.00 & 29.88 & 29.91 & 29.97 \\
0.3 & 13.10 & 13.14 & 13.10 & 13.12 & 13.17 & 13.10 & 13.11 \\
0.4 & 7.273 & 7.273 & 7.261 & 7.247 & 7.266 & 7.245 & 7.248 \\
0.5 & 4.505 & 4.478 & 4.508 & 4.505 & 4.477 & 4.489 & 4.493 \\
& & & & & & & \\
0.6 & 2.970 & 2.951 & 2.973 & 2.971 & 2.971 & 2.964 & 2.963 \\
0.7 & 2.012 & 2.000 & 2.005 & 1.994 & 2.009 & 2.004 & 2.002 \\
0.8 & 1.333 & 1.326 & 1.333 & 1.340 & 1.338 & 1.332 & 1.332 \\
0.9 & 0.7895 & 0.7918 & 0.7920 & 0.7914 & 0.7904 & 0.7911 & 0.7903 \\
\hline
\end{tabular}

\section{Interface between Anisotropic Bodies}

It is simple to extend the finite element method described in the previous sections to interfaces between two anisotropic dielectric bodies. The normalization constant $C$ appeared in the numerical result does require further treatment. To determine this constant $\mathrm{C}$, we followed the procedure of the so-called Stroh formalism that has been widely applied in linear anisotropic elasticity studies (Ting, 1995). The notation used before (Fan $(1994,1996))$ will be adopted here.

Let us start with a two-dimensional dielectric boundary value problem for general anisotropic materials, the governing equations are:

$$
\begin{aligned}
& D_{i}=\varepsilon_{i j} E_{j} \\
& E_{i}=-\phi_{, i}
\end{aligned}
$$




$$
D_{i, i}=0
$$

We can assume that the potential, $\phi$, can be considered as a function of a single complex variable,

$$
\phi=f(z)=f(x+p y)
$$

where $p$ is a complex number. By substituting Eqn.(4.4) into Eqn.(4.1), the equivalent of Eqn.(4.3) is

$$
\left(\varepsilon_{11}+2 p \varepsilon_{12}+p^{2} \varepsilon_{22}\right) \phi^{\prime}(z)=0
$$

For a non-zero solution of $\phi$, one must have

$$
\varepsilon_{11}+2 p \varepsilon_{12}+p^{2} \varepsilon_{22}=0
$$

The two roots of this equation are:

$$
p=-\frac{\varepsilon_{12}}{\varepsilon_{22}}+i \sqrt{\frac{\varepsilon_{11}}{\varepsilon_{22}}-\left(\frac{\varepsilon_{12}}{\varepsilon_{22}}\right)^{2}}
$$

and

$$
\bar{p}=-\frac{\varepsilon_{12}}{\varepsilon_{22}}-i \sqrt{\frac{\varepsilon_{11}}{\varepsilon_{22}}-\left(\frac{\varepsilon_{12}}{\varepsilon_{22}}\right)^{2}}
$$

It is noted that the $p$ must be a complex number because the permittivity tensor, $\boldsymbol{\varepsilon}$, is positive definite. In the case of isotropic bodies, $p=i$ and $-i$.

With the complex function $f(z)$, the potential and electric displacement may be rewritten as:

$$
\begin{aligned}
& \phi=2 \operatorname{Re}(f(z))=f(z)+\overline{f(z)} \\
& D_{2}=-2 \operatorname{Re}\left(L f^{\prime}(z)\right)
\end{aligned}
$$

where the bar denotes the complex conjugate in this section, and

$$
L=\varepsilon_{2 j}\left(\delta_{1 j}+p \varepsilon_{2 j}\right)=i \sqrt{\varepsilon_{11} \varepsilon_{22}-\left(\varepsilon_{12}\right)^{2}}
$$

Accordingly, the electric displacement continuity condition of Eqn.(1.2) along the interface $y=0$ is:

$$
L_{1} f_{1}^{\prime}(x)+\overline{L_{1} f_{1}{ }^{\prime}(x)}=L_{2} f_{2}{ }^{\prime}(x)+\overline{L_{2} f_{2}{ }^{\prime}(x)}
$$

where the subscripts 1 and 2 refer to the upper body and the lower body respectively. Eqn. (4.11) implies that

$$
L_{1} f_{1}^{\prime}(z)=\overline{L_{2} f_{2}{ }^{\prime}(\bar{z})} \quad \text { for } \mathrm{y}>0
$$




$$
\overline{L_{1} f_{1}{ }^{\prime}(\bar{z})}=L_{2} f_{2}{ }^{\prime}(z) \quad \text { for } \mathrm{y}<0
$$

With all these in our mind, we may have the potential discontinuity condition along the interface $y$ $=0$ as

where

$$
-i\left[\phi^{\prime}\right]=H L_{1} f_{1}^{\prime}(x)-\bar{H} L_{2} f_{2}{ }^{\prime}(x)
$$

$$
H=i\left(L_{1}^{-1}-\overline{L_{2}^{-1}}\right)
$$

Analogous to dislocation in linear elastic solids (Fan, 1994), we have

$$
L_{1} f_{1}(z)=\overline{L_{2} f_{2}(\bar{z})}=-\frac{1}{2 \pi} H^{-1}[\phi] \ln z
$$

Therefore the electric displacement along the interface can be obtained from Eqn.(4.9) as:

$$
D_{2}=\frac{1}{2 \pi}\left(H^{-1}+\bar{H}^{-1}\right) \frac{[\phi]}{x}
$$

By substituting the $H$ by Eqn.(4.14) and Eqn.(4.10), we have

$$
H^{-1}+\bar{H}^{-1}=2 H^{-1}=\frac{2 \sqrt{\varepsilon_{11}^{(1)} \varepsilon_{22}^{(1)}-\left(\varepsilon_{12}^{(1)}\right)^{2}} \sqrt{\varepsilon_{11}^{(2)} \varepsilon_{22}^{(2)}-\left(\varepsilon_{12}^{(2)}\right)^{2}}}{\sqrt{\varepsilon_{11}^{(1)} \varepsilon_{22}^{(1)}-\left(\varepsilon_{12}^{(1)}\right)^{2}}+\sqrt{\varepsilon_{11}^{(2)} \varepsilon_{22}^{(2)}-\left(\varepsilon_{12}^{(2)}\right)^{2}}}
$$

It is seen that Eqn. (4.17) becomes to the constant $C$ stated in Eqn.(2.5) when the both of the materials are isotropic, where $\varepsilon_{11}=\varepsilon_{22}$ and $\varepsilon_{12}=0$. In order words, the numerical results for the interface between two anisotropic materials will be normalized by the constant defined by Eqn. (4.17).

\section{Discussion and conclusions}

In the Fig. 3, the finite element numerical result for the plane configuration case is compared with Eqn.(2.9) which is solved via the singular equation scheme without considering the interaction among the micro cracks along the interface. It is seen that the non-interaction solution Eqn.(2.9) can be applied up to $\rho=a / b=0.3$ with less than $5 \%$ deviation from the finite element results listed in Table 1.

For the three-dimensional configuration, we studied the case of isotropic damaged interface. The isotropic assumption leads the axial symmetry configuration for our numerical analysis. It is needless to say that there is a derivation of the three-dimensional problem via the singular integral 
equation approach where elliptical function will be involved. With verified finite element analysis in plane configuration, the same mesh and procedure is applied to the axial symmetry in ABAQUS. Comparing Figure 3 and Figure 4, we see the similar trend for the two-dimensional interface and three-dimensional interface in terms of the "spring constant".

It is realized that there are other engineering problems which are described by the same set governing equations as the dielectric problem. For example, the temperature distribution function of steady heat conduction problem obeys the Laplace equation Eqn.(1.6) together with proper boundary conditions. Therefore, we can expect the imperfect interface in the heat conduction problem can be modeled via the same scheme. Another problem belongs to the same category is the imperfect interface in linear elasticity under anti-plane condition, which can be treated by the present model. Readers may realize this during the reading of our previous sections since we have borrowed the formulation and solutions from the linear elasticity.

Lastly, we would like to mention that the present boundary value problem under the self-consistent scheme is posted on a 2-dimensional surface (interface), while the original self-consistent scheme was developed on the 3-dimensional solids. It is not a simple reduction of dimensions. Rather, the level of the complexity in our self-consistent model on the 2-dimensional surface is higher than the original one, because the present model is described by a singular integral equation in nature. Sometimes, it is called mixed boundary value problem (Erdogan, 1975).

\section{Acknowledgement}

Part of the study reported here was carried out during the first author's stay at the University of Hong Kong as a William Mong Visiting Research Fellow. 


\section{References:}

1. ABAQUS (1998) user's manual, Version 5.8, Hibbitt, Karlsson \& Sorensen, Rhode Island, USA.

2. Benveniste, Y. (1999), " On the decay of end effects in conduction phenomena: A sandwich strip with imperfect interfaces of low or high conductivity”, Journal of Applied Physics, Vol86, No.3 pp1273-1279.

3. Benveniste, Y. and Milho, T. (1999), "Neutral inhomogeneities in conduction phenomena", Journal of the Mechanics and Physics of Solids, Vol.47, pp1873-1892.

4. Christensen R.M. (1990), “A critical review for a class of micro-mechanics models”, Journal of the Mechanics and Physics of Solids, Vol.38, No.3, pp379-404.

5. Erdogan, F. (1975), "Mixed boundary value problems in mechanics" ed. by S. Nemat-Nasser, Mechanics Today pp1-86.

6. Fan H, Han Z. and Sze K.Y. (2000), "Micro-mechanics modeling for asperity contact", submitted to Mechanics of Materials.

7. Fan, H. (1995),"Decay rates in a piezoelectric strip", International Journal of Engineering Science. Vol.33, No.8, pp1095-1103.

8. Fan, H., (1994), "Interfacial Zener-Stroh crack", ASME Journal of Applied Mechanics, Vol.61, No.4, pp829-834.

9. Hashin, Z. (1991), “The spherical inclusion with imperfect interface”, ASME Journal Applied Mechanics, Vol.58, No.3 pp444-449.

10. Margetan, F.J. Thompson, R.B. and Gray T.A. (1988) "Interfacial spring model for ultrasonic interactions with imperfect interfaces" Journal of Nondestructive Evaluation. Vol.7, No.3/4, pp131-152.

11. Milho, T. and Benveniste, Y. (1999), "On the effective conductivity of composites with ellipsoidal inhomogeneities and high conducting interface”, Proc. Roy. Soc. Lond. A. 455pp2687-2706.

12. Suo, Z. Kuo, C.M. Barnett, D.M. and Willis J.R., (1991) “Fracture Mechanics for piezoelectric ceramics", Journal of the Mechanics and Physics of Solids, Vol.40, pp739-765.

13. Ting, T.C.T. (1995), Anisotropic Elasticity, Oxford University Press, UK 


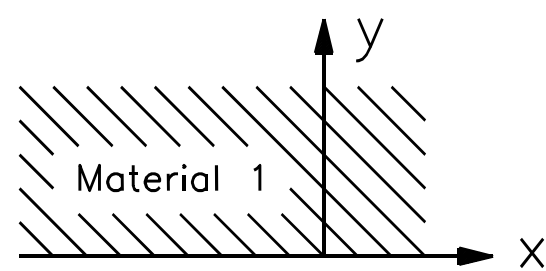

Material 2

Figure 1(a). A bimaterial interface

Figure 1(b). An imperfect interface with microcracks
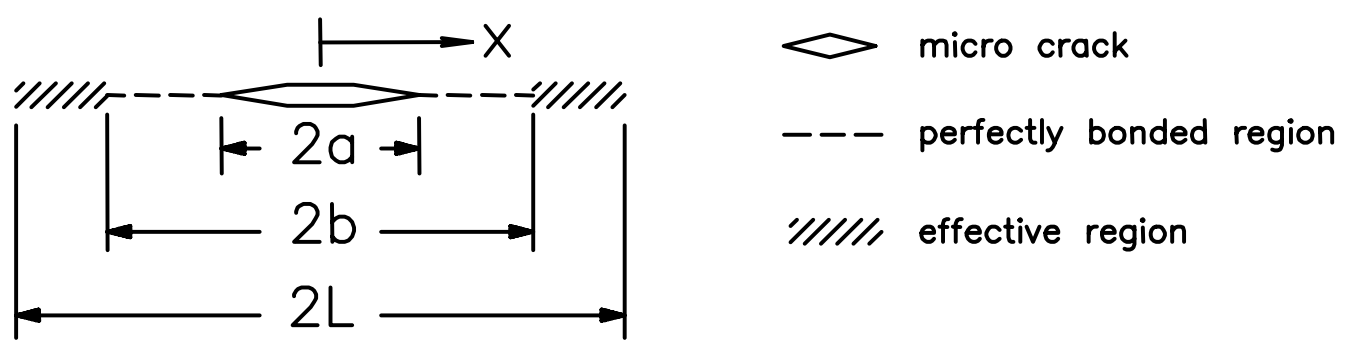

Figure 1(c). A self-consistent model for the interface in Figure 1(b). 


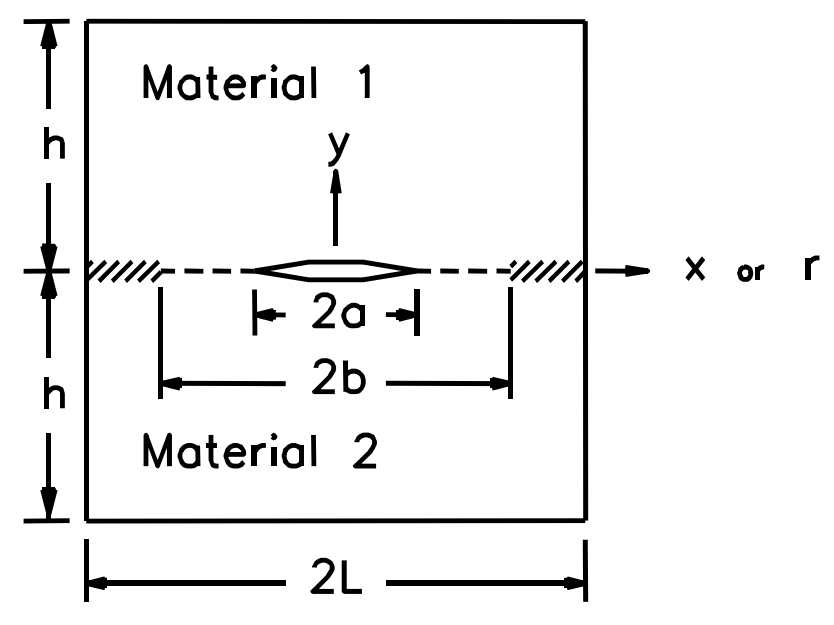

Figure 2. Problem domain for finite element solutions. Problem parameters :

$$
b=1, h=L=15, \varepsilon^{(1)}=1, a \text { and } \varepsilon^{(2)} \text { are varied }
$$

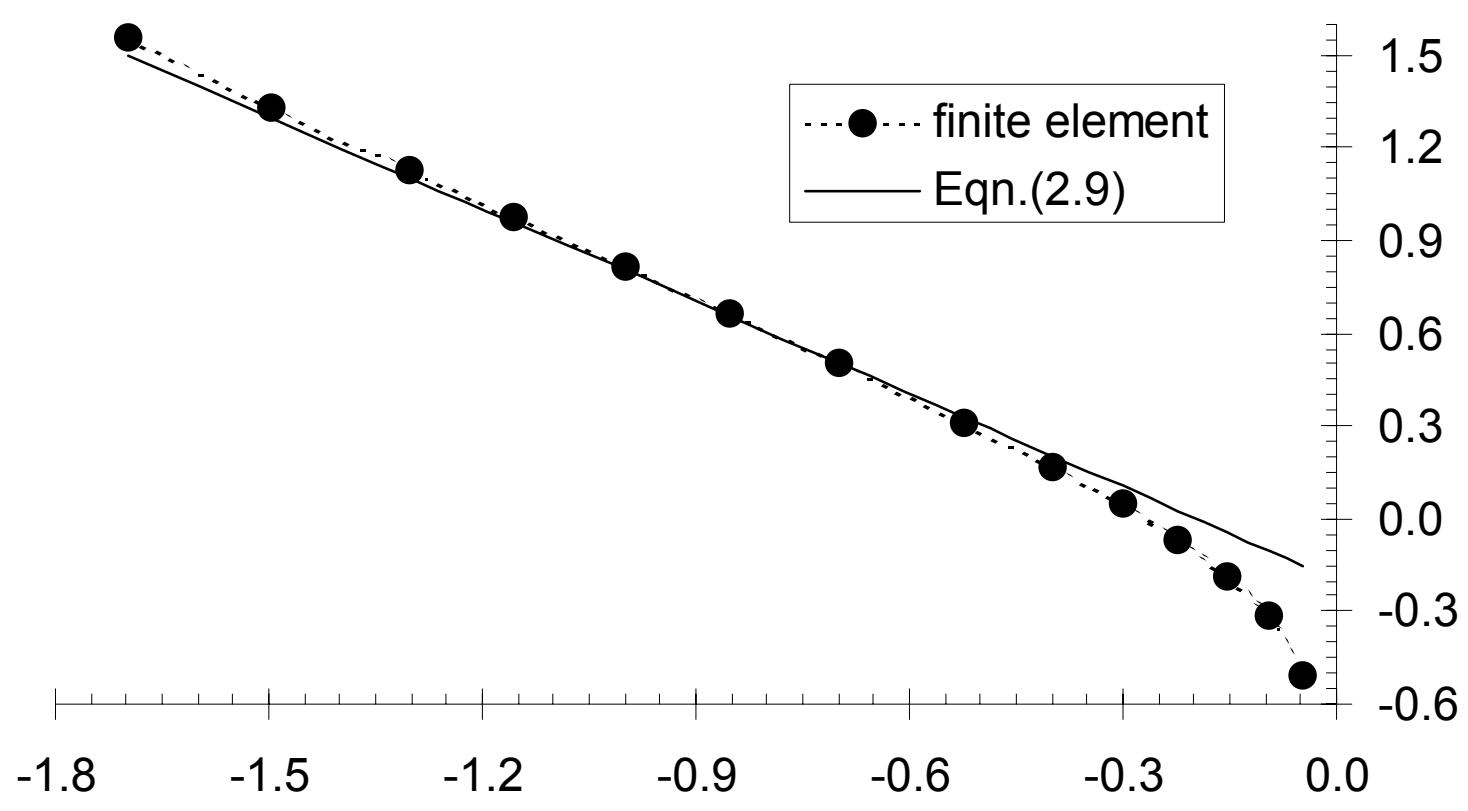

Figure 3. $\log (k a / C)$ versus $\log (a / b)$ for plane configuration obtained by finite element computation and Eqn.(2.9) 


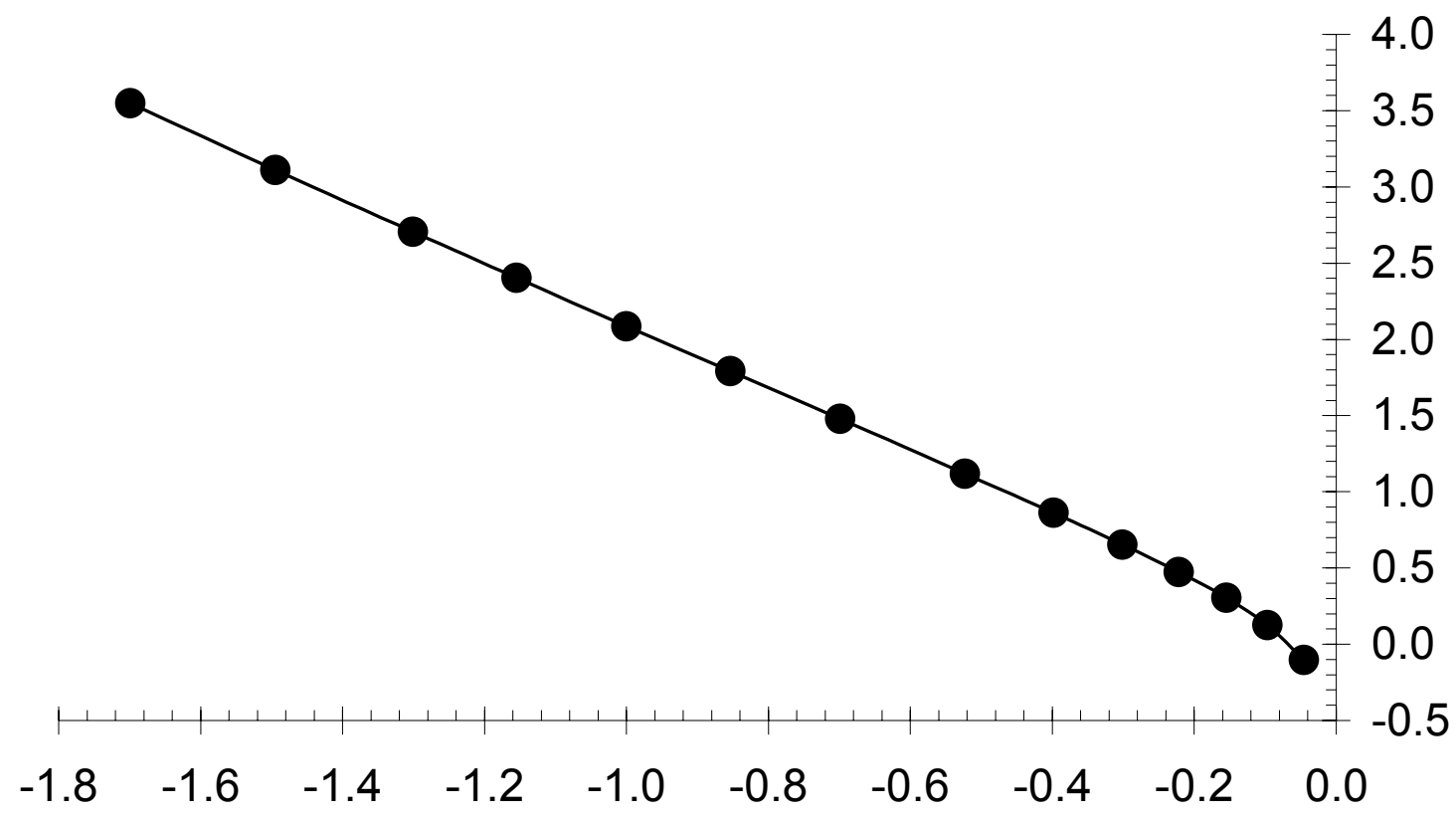

Figure 4. $\log (k a / C)$ versus $\log (a / b)$ for axisymmetric configuration obtained by finite element computation 